\section{Endangered Spanish science}

SIR - We should like to respond to Javier Garcia-Guinea (Nature 379, 109; 1996). He says there are two kind of scientists in Spain, those who publish in journals ranked in the Science Citation Index (SCI) and what he calls "parallel scientists". He complains that the latter are denied grants or receive less funding and respect than their counterparts. And he says that they "... publish in serious Spanish journals that are not in the $S C I$ lists. They often know French and German (rather than English) and carry out other activities such as teaching, writing books, organizing meetings or running laboratories."

Most of the "serious" Spanish journals are not refereed journals in the accepted sense; the publisher, editorial board, referees and authors belong to the same small group of people. What makes a good journal is not whether it is written in English or Spanish but that publication is restricted to high quality papers.

As far as the language itself is concerned, for all the disciplines Garcia mentions, the most important papers and the leading journals are published in English. So how can researchers who cannot understand English know what is happening in their fields? And how can they communicate their exciting findings to the international scientific com- munity? Science in the twentieth century consists not only of gathering exciting data or doing good experiments, but sharing the results in a common effort. We understand the deficiencies in the Spanish educational system, which only lately has been able to acknowledge the importance of English as the official language of science; true ignorance comes not from not knowing something but from not recognizing the need to learn.

Finally, the SCI scientists also teach (although perhaps less than the parallel ones), write books or book chapters (most of them in English, internationally distributed), organize international meetings and run laboratories (which are increasingly joined by researchers from other countries).

In Spain, as in any other country, there are two kinds of scientists, bad and good. Many of us are training abroad, trying to do quality research and facing now the uncertainties of an increasingly saturated job market. Many of us have tried to join Spanish institutions only to find the doors closed because we publish in $S C I$ journals but do not have teaching experience. (The undergraduate and graduate training that we supervise counts for nothing.) Many of us know nobody among the parallel scientists who still govern many Spanish institutions, and thus need to live with our heart in one place and our brains and hands in another. For us, the hope that mediocrity will be banished from Spanish science and that quality criteria will direct scientific policies is the only thing left.

\section{Rosa Carballada}

Cristina Hernández-Munain

Carlos Suñe-Negre

Duke University Medical Center,

Durham, North Carolina 27710, USA

SIR - Recent correspondents have drawn attention of the problems of Spanish scientists and the uncertain future. This is a sad paradox : between 1982 and 1992, "the rise in scientific production in Spain has been disproportionately greater than in the rest of the world" (F. J. Ayala, Politica Científica, 43, 5-12; 1995). This increase has been largely the work of young scientists with postdoctoral training in foreign institutions. They are now returning to Spanish institutes under 3-year contracts to carry out particular tasks within continuing projects. Some 600 young scientists now have these 3 -year contracts. They may account for 20-30 per cent of researchers in some flagship institutes of the Spanish Council for Scientific Research (CSIC).

Because the first opportunity to hire postdocs arose in 1992, some of these highly qualified researchers have now finished their contracts and are facing an uncertain

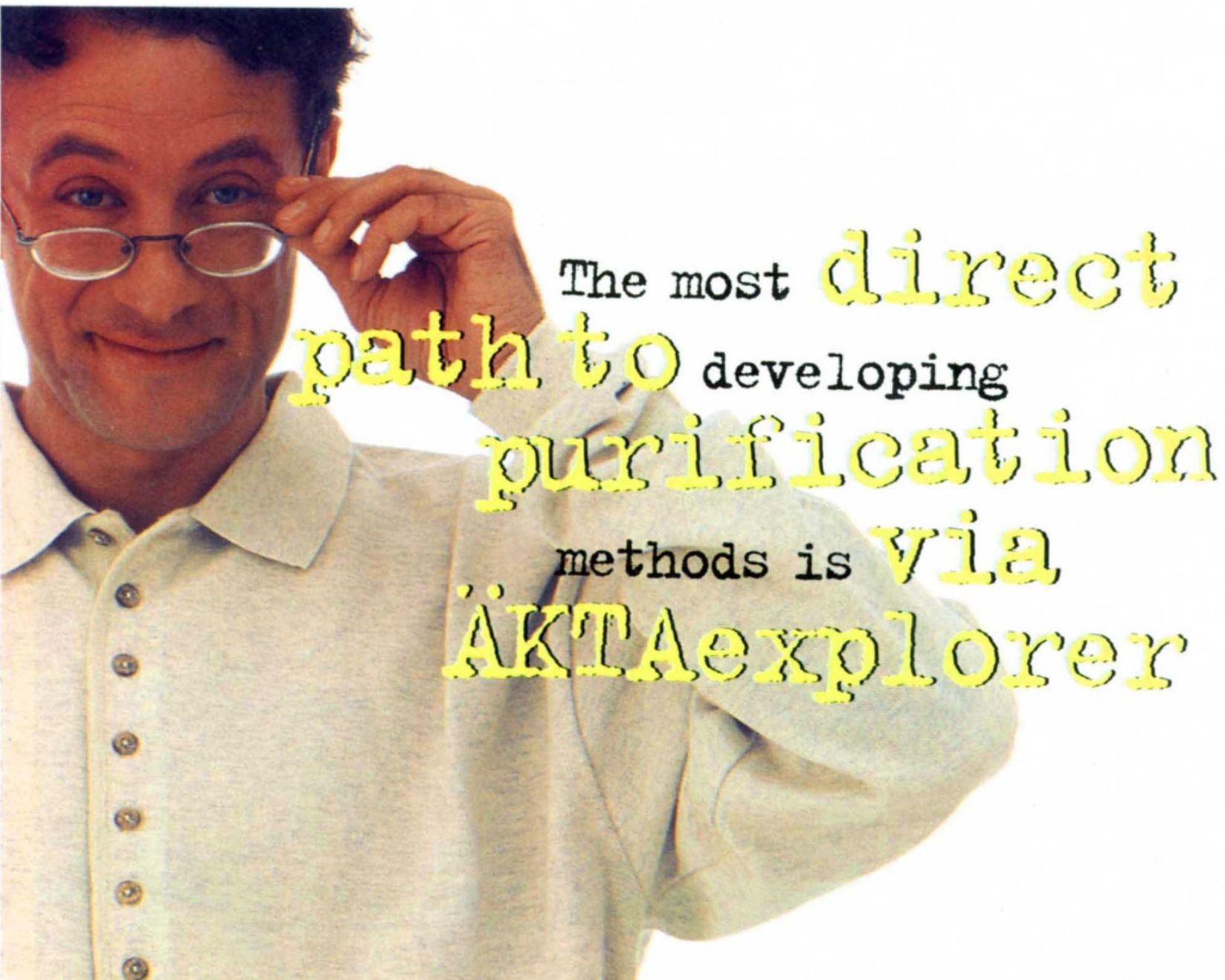


future. Some of them have chosen to go abroad again as postdocs, others have abandoned research after almost a decade, and many are joining the ranks of the jobless. The reason? There is a shortage of research posts. In the last opening for CSIC faculty, only 50 staff positions were available for the whole of Spain. That has been the situation for the past 3 years. Between 1982 and 1992, the amount invested in science increased from 0.48 to 0.9 per cent of Spain's gross domestic product (GDP); during the same period, the $S C I$ impact factors of papers from Spain increased from 1.2 to 1.78.

Now, however, the same scientific authorities have failed to follow through. For most of us, these contracts were considered a springboard to an independent position. In the event, the conditions under which we were taken on preclude us from working on any other project. We can neither submit proposals for funding to national boards (such as the Interministry Committee of Science and Technology; CICYT), nor to any other institutions. So our hands are tied. Only staff scientists can apply for funds or be principal researchers on a project. Moreover, there are no tenure-track positions available. Funds for science need to grow to at least 2 per cent of GDP (as in other European Union countries) with a corresponding increase in the number of researchers (currently 1.4 per 1,000 , compared with 1.9 per 1,000 else- where in the European Union).

The future of Spanish science depends on a proper solution to the problems faced by these returned scientists, who can help to obtain the benefits expected from the past decade's effort and obtain funds in fair competition for international projects with other European colleagues and institutions.

Juan F. Beltrán

Juan J. Negro

José L. Yela

José A. Mateo

Estación Biológica de Doñana, CSIC, Apdo 1056,

41080 Sevilla, Spain

\section{Study in Germany}

SIR - A recent leading article (Nature 378, 755 ; 1995) mentions a European Commission (EC) survey of young European scientists who applied for fellowships to train in another European Union country under the EC's Human Capital and Mobility (HCM) programme: only 10 per cent out of 6,000 applied to do research in Germany. You interpret these figures as a sign of "the unpopularity of Germany as a place for young foreign scientists".

I should like to point out that Germany, because it has a national sponsoring scheme to attract young foreign scientists, is less dependent on the new European pro- gramme than other countries in Europe. Under existing programmes of the Alexander von Humboldt Foundation, the German Academic Exchange Service, the Helmholtz Association of National Research Centres and the Max Planck Society, more than 1,500 young foreign scientists and scholars from other European Union countries came to German research establishments in 1994. The Humboldt Foundation alone registered 40 per cent more applications than ten years ago. The increase in real sponsorship figures for the Max Planck Society was 80 per cent in 10 years.

Young foreign scientists are most welcome in Germany. English is a current language in many institutes and laboratories. German language courses are available in all national programmes. Many guest researchers have told us that German is not a difficult language to learn. Foreign guests are as a rule exempted from the burden of bureaucracy in universities. We are not aware of any hostility towards foreigners in universities. On the contrary, cooperation with foreign guests is seen as an enrichment for national research. We are grateful, therefore, for the positive conclusion to your leading article.

\section{Manfred Osten}

(Secretary General)

Alexander von Humboldt Foundation,

Jean-Paul-Str. 12,

D-53173 Bonn, Germany
If you've ever developed a purification strategy by yourself, you know there is a lot to consider. Creating media screening schemes; designing buffer preparation routines; selecting which columns to use - even listing the tasks takes careful consideration. But now there's a better way of working. screening. You'll save time as the system automatically recommends the best columns for your runs. You get fast $\mathrm{pH}$ screening as it automatically prepares your buffers from stock solutions. The moment you turn ÄKTAexplorer on, you're presented with a direct path to full-scale purification.

That path is UNICORN ${ }^{\circledR}$ - ÄKTAexplorer's control software. Your scale-up is simplified as the system's software can transfer and implement your methods on purification systems at all scales.

Of course, these are just a few of ÄKTAexplorer's features. So call us at I (800) 5263593 from North America, +8I (0)3 34926949 from Japan or $+46(0) 18 \quad 1650 \quad 11$ from the rest of the world (or meet us on the Internet at http://www.biotech.pharmacia.se/akta.htm). scouting and media

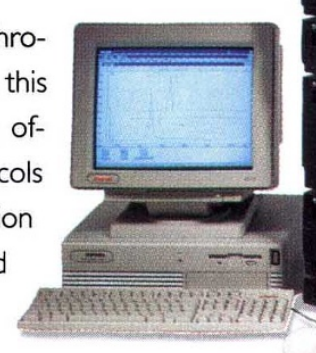

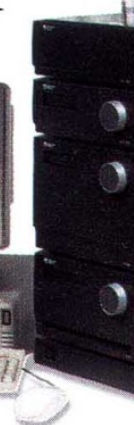

,

Turn ÄKTA"wexplorer on, choose a protocol, check running parameters
explorer does the rest.

matographic techniques, this new purification system offers you pre-set protocols for every major purification

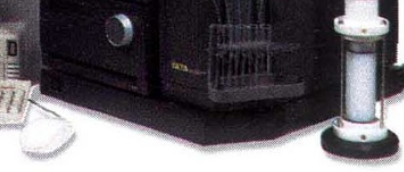

